\title{
Remarks on Central Limit Theorems for the Number of Percolation Clusters
}

\author{
By \\ Nobuaki Sugimine* and Masato TAKeI**
}

\begin{abstract}
We consider percolation problems on regular trees and some pre-fractal graphs. Central limit theorems for the number of open clusters in a nite box are obtained. For regular trees and some classes of Sierpinski carpet lattices, we can prove the central limit theorems for all $p \in\left(\begin{array}{ll}0 & 1\end{array}\right)$.
\end{abstract}

\section{$\S 1 . \quad$ Introduction and Results}

Central limit theorems (CLT's) for percolation problems have been studied by many authors (see Grimmett [2] §11.6). For Bernoulli bond percolation problem on $\mathbb{Z}^{d}(d \geq 2)$, Zhang [9] proved a CLT for the number of open clusters in a finite box for all $p \in(0,1)$, that is, including the case $p=p_{c}\left(\mathbb{Z}^{d}\right)$. His proof is based on McLeish's martingale CLT [5]. Using this method together with the ergodic theorem, Penrose [6] proved a general CLT which can be applied to several models. In this note, by using the argument in [9] we study a CLT for percolation problems on regular trees and Sierpiński carpet lattices, where the ergodic theorem is not available.

Communicated by Y. Takahashi. Received June 4, 2004. Revised November 11, 2004. 2000 Mathematics Subject Classification(s): Primary 82B43; secondary 60F05, 28A80. Key words: percolation, central limit theorem, tree, Sierpiński carpet.

*Aihara Complexity Modelling Project, ERATO, JST, 45-18 Oyama-cho, Shibuya-ku, Tokyo 151-0065, Japan.

e-mail: sugimine@aihara.jst.go.jp

** Department of Information and Media Science, Graduate School of Science and Technology, Kobe University, Rokko, Kobe 657-8501, Japan.

e-mail: takei@math.kobe-u.ac.jp

Current address: Department of Mathematics, Faculty of Science and Technology, Keio University, 3-14-1 Hiyoshi, Kohoku-ku, Yokohama 223-8522, Japan.

e-mail: takei@math.keio.ac.jp

(C) 2006 Research Institute for Mathematical Sciences, Kyoto University. All rights reserved. 


\section{$\S 1.1$. Bernoulli bond percolation}

Let $G=(V, E)$ be an infinite connected graph. We fix an arbitrary point as the origin. Each bond $e \in E$ is independently declared to be open with probability $p$ and closed with probability $1-p$. We denote the Bernoulli measure on $\{\text { open, closed }\}^{E}$ by $P_{p}$. The expectation, the variance and the covariance relative to $P_{p}$ are denoted by $E_{p}, \operatorname{var}_{p}$ and $\operatorname{cov}_{p}$, respectively. The open cluster containing the origin is denoted by $C$. The percolation probability $\theta(p)=$ $P_{p}(|C|=\infty)$ is an increasing function in $p$, where $|C|$ denotes the number of vertices in $C$. We define the critical probability $p_{c}(G)=\sup \{p ; \theta(p)=0\}$. We fix an increasing sequence $\{B(n)\}$ of finite regions containing the origin. Let

$$
K_{n}=\sum_{x \in B(n)} \frac{1}{\left|C_{n}(x)\right|} \text { and } \tilde{K}_{n}=\sum_{x \in B(n)} \frac{1}{\left|C_{n}(x)\right|} 1_{\left\{\left|C_{n}(x)\right|>1\right\}}
$$

where $C_{n}(x)=\{y \in B(n)$; there is an open path in $B(n)$ from $x$ to $y\}$. In other words, when we regard isolated points as clusters, we denote the number of open clusters in $B(n)$ by $K_{n}$. Otherwise we denote it by $\tilde{K}_{n}$.

We say that the central limit theorem holds for $\left\{f_{n}\right\}$ if

$$
\frac{f_{n}-E_{p} f_{n}}{\sqrt{\operatorname{var}_{p} f_{n}}} \stackrel{\mathcal{L}}{\longrightarrow} \mathcal{N}(0,1) \text { as } n \rightarrow \infty,
$$

where $\stackrel{\mathcal{L}}{\longrightarrow}$ denotes the convergence in law and $\mathcal{N}(0,1)$ denotes the standard normal distribution.

\section{§1.2. Regular trees $\mathbb{T}^{d}$}

For an integer $d \geq 2$, let $\mathbb{T}^{d}=\left(V^{d}, E^{d}\right)$ be the infinite connected graph containing no cycles in which each vertex has $(d+1)$ edges. We fix a point as the origin, denoted by $O \in V^{d}$.

Theorem 1.1. We consider the Bernoulli bond percolation problem on $\mathbb{T}^{d}(d \geq 2)$. Let $B(n)=\{x ; d(O, x) \leq n\}$, where $d(\cdot, \cdot)$ denotes the graph distance. For any $p \in(0,1)$, the central limit theorems for $\left\{K_{n}\right\}$ and $\left\{\tilde{K}_{n}\right\}$ hold.

Some remarks are in order. We note that the CLT for $\left\{K_{n}\right\}$ follows from the CLT for i.i.d. sequences, using the geometry of trees: we can see that

$$
K_{n}=|B(n)|-\left.|| \omega\right|_{B(n)}||=\left.\|B(n)||+1-\| \omega\right|_{B(n)} \|,
$$


where $\|\cdot\|$ denotes the number of the bonds. It is well-known that there are infinitely many infinite clusters when $p>p_{c}\left(\mathbb{T}^{d}\right)$ (see e.g. [3]) while the uniqueness of the infinite cluster plays an important role in [9]. But we do not need the uniqueness for proving the CLT, due to the geometric character of trees. We can obtain the CLT for the number of open clusters in Bernoulli site percolation by a similar proof as for $\tilde{K}_{n}$. Our proof is valid for trees for which we can verify (2.3) and (2.4), e.g. trees of bounded degree.

\section{$\S 1.3$. Sierpiński carpet lattices $G_{T}$}

Generalized Sierpiński carpets. Let $L \geq 2$. For $0 \leq i_{1}, i_{2} \leq L-1$, let $\Psi_{\left(i_{1}, i_{2}\right)}: \mathbb{R}^{2} \rightarrow \mathbb{R}^{2}$ be an affine map which maps $[0,1]^{2}$ to $\left[i_{1} / L,\left(i_{1}+1\right) / L\right] \times$ $\left[i_{2} / L,\left(i_{2}+1\right) / L\right]$, preserving the directions. For $T \subset \mathbf{T}_{L} \equiv\{0, \ldots, L-1\}^{2}$, there exists a unique compact set $K^{T} \subset[0,1]^{2}$ such that $K^{T}=\bigcup_{t \in T} \Psi_{t}\left(K^{T}\right)$. This is called a generalized Sierpiński carpet.

Sierpiński carpet lattices. Hereafter we assume that $(0,0) \in T$ and $K^{T}$ is connected. Let

$$
F_{n}^{T}=\bigcup_{t_{1}, \ldots, t_{n} \in T} \Psi_{t_{1}} \circ \cdots \circ \Psi_{t_{n}}\left([0,1]^{2}\right) .
$$

A sequence of graphs $G_{n}^{T}=\left(V_{n}^{T}, E_{n}^{T}\right)(n=1,2, \ldots)$ are defined by

$$
V_{n}^{T}=L^{n} F_{n}^{T} \cap \mathbb{Z}^{2}, E_{n}^{T}=\left\{\langle u, v\rangle ; u, v \in V_{n}^{T},|u-v|=1\right\},
$$

where $|\cdot|$ denotes the usual Euclidean norm. The graph $G_{T}=\left(V_{T}, E_{T}\right)$ which is defined by

$$
V_{T}=\bigcup_{n=1}^{\infty} V_{n}^{T}, E_{T}=\bigcup_{n=1}^{\infty} E_{n}^{T}
$$

is called the Sierpiński carpet lattice corresponding to $K^{T}$.

We consider the bond percolation on $G_{T}$. Shinoda [7] obtained a sufficient condition for $T$ to satisfy $p_{c}\left(G_{T}\right)<1$. However, since $G_{T}$ is in general not periodic, it is difficult to know further properties, e.g. whether the infinite cluster is unique or not.

Example 1. When $L=3$ and $T=\mathbf{T}_{3} \backslash\{(1,1)\}, K^{T}$ is the Sierpiński carpet. We call corresponding $G_{T}$ the pre-Sierpiński carpet (see Figure 1). It is known that $p_{c}\left(G_{T}\right)<1$ (see below). Using the rescaling argument in [1], which can be applied to higher dimensional cases, $\mathrm{Wu}[8]$ proved the uniqueness of the infinite cluster when $p$ is sufficiently close to 1 . 


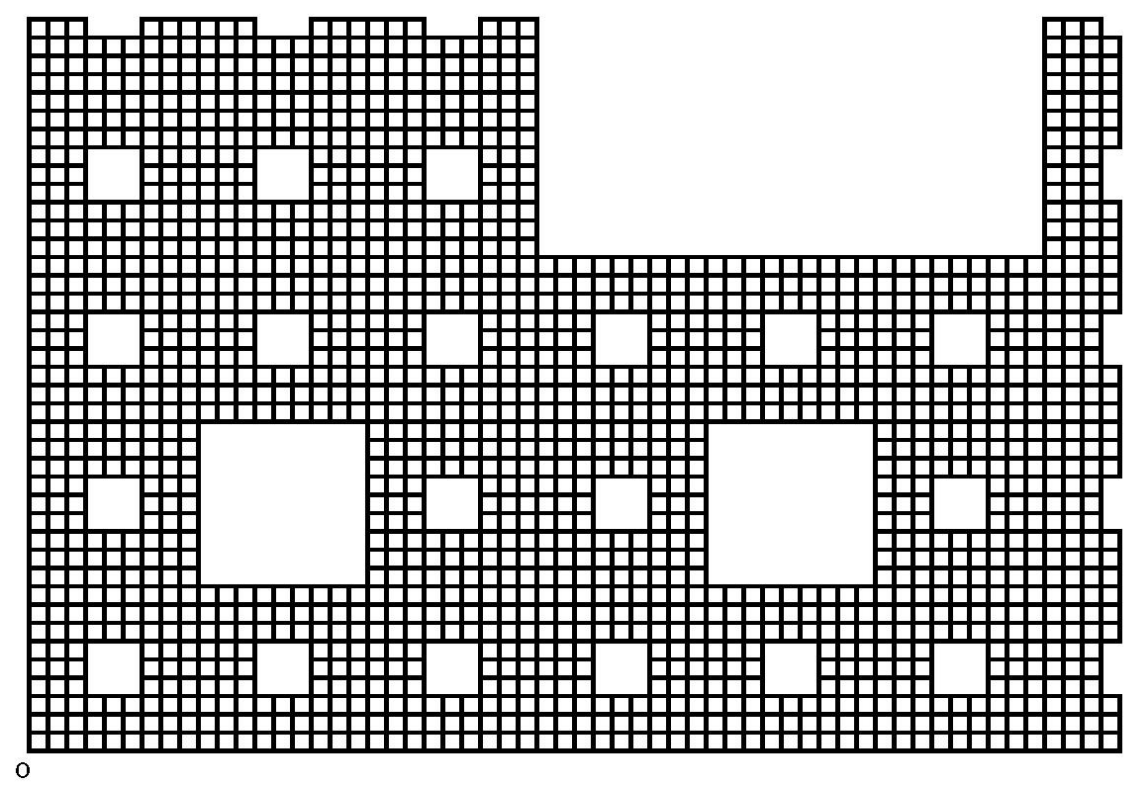

Figure 1. The pre-Sierpiński carpet $\left(G_{T}\right.$ with $\left.T=\mathbf{T}_{3} \backslash\{(1,1)\}\right)$.

Here we treat a class of planar Sierpiński carpet lattices, which was considered by Kumagai [4]. We consider the following conditions for $T \subset \mathbf{T}_{L}$ :

$$
\begin{aligned}
& K^{T} \text { is connected. } \\
& (i, j) \in T \Longrightarrow(j, i) \in T,(i, L-1-j) \in T . \\
& \{(0, j) ; 0 \leq j \leq L-1\} \subset T .
\end{aligned}
$$

We consider sponge percolation problems on $G_{T}$. Hereafter we sometimes omit $T$ and we write $G_{n}$ also for the graph congruent to the "original" $G_{n}=G_{n}^{T}$. Let $G_{n,[l, m]}$ be the rectangle which is defined by placing $m G_{n}$ 's horizontally and $l G_{n}$ 's vertically. We also consider a dual graph $G_{n,[l, m]}^{*}$ of $G_{n,[l, m]}$ (see Figures 2 and 3 . The precise definition is found in [4]). We define the following crossing probabilities;

$$
\begin{aligned}
& Q_{n,[l, m]}(p) \\
& \quad=P_{p}\left(\text { there is an open crossing from the bottom to the top in } G_{n,[l, m]}\right), \\
& Q_{n,[l, m]}^{*}(p) \\
& \quad=P_{p}\left(\text { there is an closed crossing from the bottom to the top in } G_{n,[l, m]}^{*}\right) .
\end{aligned}
$$




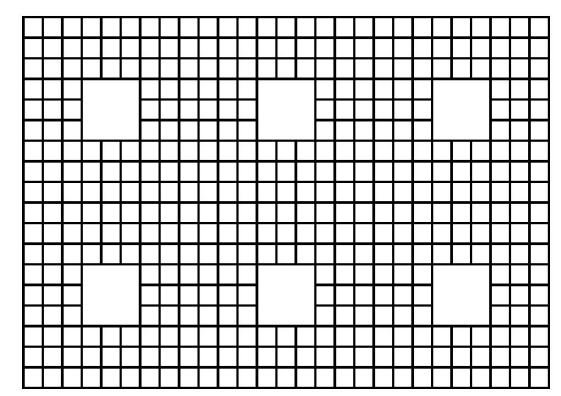

Figure 2. Sponge $G_{2,[2,3]}$.

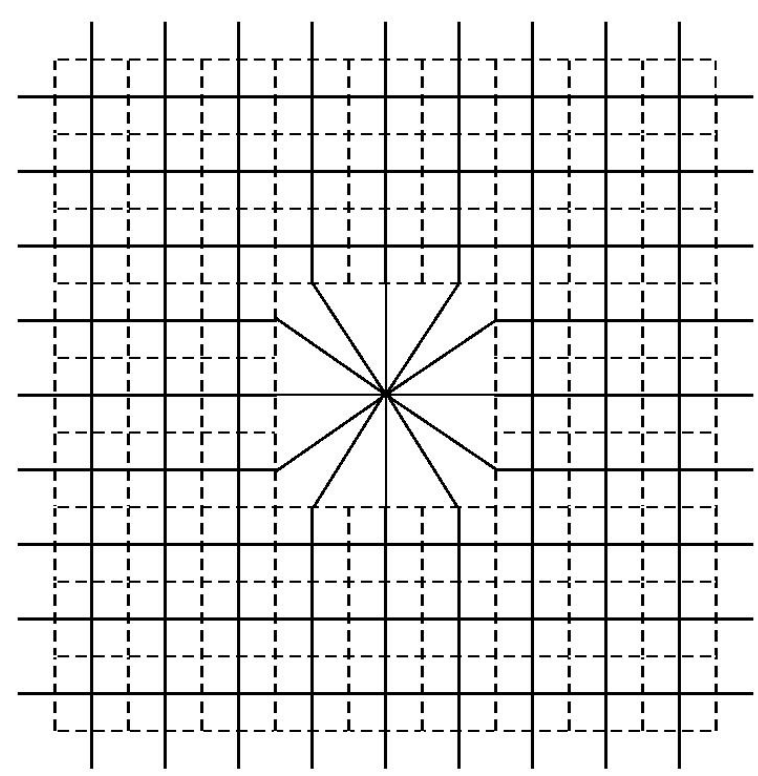

Figure 3. 'Dual sponge' $G_{2,[1,1]}^{*}$.

Note that $Q_{n,[l, m]}(p)+Q_{n,[m, l]}^{*}(p)=1$. We define the following critical points;

$$
p_{s}=\sup \left\{p ; \limsup _{n \rightarrow \infty} Q_{n,[1,3 L]}(p)=0\right\}, p_{s}^{*}=\inf \left\{p ; \limsup _{n \rightarrow \infty} Q_{n,[1,3 L]}^{*}(p)=0\right\} .
$$

If $T \subset \mathbf{T}_{L}$ satisfies (1.1), (1.2) and (1.3), then $0<p_{s} \leq p_{c} \leq p_{s}^{*}<1$ ([4]). Kumagai [4] proved that $p_{s}=p_{c}=p_{s}^{*}, \theta\left(p_{c}\right)=0$ and the uniqueness of the 
infinite cluster for $p>p_{c}$, under the condition

$$
\sup \left\{p ; \limsup _{n \rightarrow \infty} Q_{n,[3 L, 2]}(p)<1\right\}=\sup \left\{p ; \limsup _{n \rightarrow \infty} Q_{n,[3 L, 1]}(p)<1\right\}\left(=p_{s}^{*}\right),
$$

which is important for studying the critical regime. It is noted in [4] that (1.4) holds if $T$ satisfies (1.1), (1.2) and $\{(0, j),(1, j) ; 0 \leq j \leq L-1\} \subset T$.

Theorem 1.2. We assume that $T$ satisfies (1.1), (1.2) and (1.3). We consider Bernoulli bond percolation on a Sierpinski carpet lattice $G_{T}$. Let $B(n)=G_{n}^{T}$ (with some abuse of notation).

(i) For all $p \in[0,1]$, the limit $m(p) \equiv \lim _{n \rightarrow \infty} \frac{E_{p} K_{n}}{\|B(n)\|}$ exists and

$$
\lim _{n \rightarrow \infty} \frac{K_{n}}{\|B(n)\|}=m(p) \text { a.s. }
$$

(ii) We can prove the CLT for $\left\{K_{n}\right\}$ for $p \in(0,1) \backslash\left[p_{s}, p_{s}^{*}\right]$. Moreover, if (1.4) is satisfied, then the CLT for $\left\{K_{n}\right\}$ holds for all $p \in(0,1)$.

We remark that similar results can be proved for $\left\{\tilde{K}_{n}\right\}$. Our proof of the above CLT for $p \in\left(0, p_{s}\right)$ is based on the fact that $Q_{n,[1,3]}(p) \rightarrow 0$ as $n \rightarrow \infty$ (see $\S 3.3)$. Even if $T$ does not satisfy (1.1), (1.2) or (1.3), we obtain the CLT for $p$ when we can show that suitable analogues of $Q_{n,[1,3]}(p) \rightarrow 0$ as $n \rightarrow \infty$. We give some examples.

Example 2. When $L=2$ and $T=\mathbf{T}_{2} \backslash\{(1,1)\}, K^{T}$ is the Sierpiński gasket. We call corresponding $G_{T}$ (a variant of) the pre-Sierpiński gasket. Since $K^{T}$ is a finitely-ramified fractal, it is easily checked that $p_{c}\left(G_{T}\right)=1$. Since $T$ has a reflection symmetry and $Q_{n,[1,3]}(p) \rightarrow 0$ as $n \rightarrow \infty$, we can prove the CLT for $p \in(0,1)$ by using the argument in $\S 3.3$.

Example 3. Let $L=2 l+1$ with $l \geq 1$ and

$$
T=\{(0, j),(L-1, j),(j, l) ; 0 \leq j \leq L-1\}
$$

Since $T$ is anisotropic, we have to consider both left-right and top-bottom crossing probabilities. While $K^{T}$ is an infinitely-ramified fractal, it is known that these crossing probabilities tend to zero as $n \rightarrow \infty([4,7])$. Thus we can obtain the CLT for $p \in(0,1)$. 


\section{§2. Proof for Regular Trees}

Our proof is based on the argument in [9]. We enumerate the elements of $E^{d}$ as $e_{1}, e_{2}, \ldots$ according to the following rule:

Let $A(n)=B(n) \backslash B(n-1)$, where $B(0)=\emptyset$.

1) If $m<n$, then for any $e_{i} \in A(m)$ and $e_{j} \in A(n)$ we have $i<j$.

2) For any $i,\left\{e_{1}, e_{2}, \ldots, e_{i}\right\}$ is connected.

Let $q_{n}=\|B(n)\|$. We write $\Omega=\{0,1\}^{E^{d}} \ni \omega=\left(\omega_{1}, \omega_{2}, \ldots\right)$. We define $\sigma$-fields $\mathcal{F}_{0}=\{\emptyset, \Omega\}$ and $\mathcal{F}_{k}=\sigma\left[\omega_{1}, \ldots, \omega_{k}\right](k \geq 1)$. Let $f_{n}=K_{n}$ or $\tilde{K}_{n}$. Noting that $f_{n}$ depends only on the first $q_{n}$ coordinates, we can write

$$
f_{n}-E_{p} f_{n}=\sum_{k=1}^{q_{n}}\left\{E_{p}\left[f_{n} \mid \mathcal{F}_{k}\right]-E_{p}\left[f_{n} \mid \mathcal{F}_{k-1}\right]\right\}
$$

Let $\Delta_{k, n}=E_{p}\left[f_{n} \mid \mathcal{F}_{k}\right]-E_{p}\left[f_{n} \mid \mathcal{F}_{k-1}\right]\left(1 \leq k \leq q_{n}\right)$. These are martingale differences : $E_{p}\left[\Delta_{k, n} \mid \mathcal{F}_{k-1}\right]=0$. This implies that $\operatorname{var}_{p} f_{n}=\sum_{k=1}^{q_{n}} E_{p} \Delta_{k, n}^{2}$. Thus we have

$$
\frac{f_{n}-E_{p} f_{n}}{\sqrt{\operatorname{var}_{p} f_{n}}}=\sum_{k=1}^{q_{n}} X_{k, n}, \text { where } X_{k, n}=\frac{\Delta_{k, n}}{\sqrt{\sum_{k=1}^{q_{n}} E_{p} \Delta_{k, n}^{2}}} .
$$

Since $\Delta_{k, n}(\omega)$ is $\mathcal{F}_{k}$-measurable, we regard $\Delta_{k, n}(\omega)$ as a function of the first $k$ coordinates of $\omega$. We have

$$
\begin{aligned}
& \Delta_{k, n}\left(\omega_{1}, \ldots, \omega_{k-1}, \alpha\right) \\
& =\sum_{\omega_{k+1}^{\prime}, \ldots, \omega_{q_{n}}^{\prime}=0,1} \delta_{k} f_{n}\left(\omega_{1}, \ldots, \omega_{k-1}, \alpha, \omega_{k+1}^{\prime}, \ldots, \omega_{q_{n}}^{\prime}\right) \\
& \quad \times P_{p}\left\{\left(\omega_{k+1}, \ldots, \omega_{q_{n}}\right)=\left(\omega_{k+1}^{\prime}, \ldots, \omega_{q_{n}}^{\prime}\right)\right\},
\end{aligned}
$$

where $\alpha \in\{0,1\}$ and

$$
\begin{aligned}
\delta_{k} & f_{n}\left(\omega_{1}, \ldots, \omega_{k-1}, \alpha, \omega_{k+1}^{\prime}, \ldots, \omega_{q_{n}}^{\prime}\right) \\
= & p^{1-\alpha}(1-p)^{\alpha}\left\{f_{n}\left(\omega_{1}, \ldots, \omega_{k-1}, \alpha, \omega_{k+1}^{\prime}, \ldots, \omega_{q_{n}}^{\prime}\right)\right. \\
& \left.\quad-f_{n}\left(\omega_{1}, \ldots, \omega_{k-1}, 1-\alpha, \omega_{k+1}^{\prime}, \ldots, \omega_{q_{n}}^{\prime}\right)\right\} .
\end{aligned}
$$

We will check that $\left\{X_{k, n}\right\}$ satisfy the conditions of McLeish's martingale CLT ([5] Theorem 2.3): 
(a) $\max _{1 \leq k \leq q_{n}}\left|X_{k, n}\right|$ is bounded under $L^{2}$-norm, uniformly over $n$.

(b) $\max _{1 \leq k \leq q_{n}}\left|X_{k, n}\right| \stackrel{\mathrm{p}}{\longrightarrow} 0$ as $n \rightarrow \infty$.

(c) $\sum_{k=1}^{q_{n}} X_{k, n}^{2} \stackrel{\mathrm{p}}{\longrightarrow} 1$ as $n \rightarrow \infty$.

Here $\stackrel{\mathrm{p}}{\longrightarrow}$ denotes the convergence in probability. To verify the conditions (a) and (b), it is sufficient to check (2.2) and (2.3):

(2.2) There exists $M>0$ such that $\left|\Delta_{k, n}\right| \leq M$ for all $n$ and $k$.

$$
\text { There exists } \sigma=\sigma(p)>0 \text { such that } \sum_{k=1}^{q_{n}} E_{p} \Delta_{k, n}^{2} \geq \sigma q_{n} \text { for all } n \text {. }
$$

To prove (c), we have only to show that

$$
\frac{1}{q_{n}} \sum_{k=1}^{q_{n}}\left(\Delta_{k, n}^{2}-E_{p} \Delta_{k, n}^{2}\right) \stackrel{\mathrm{p}}{\longrightarrow} 0 \text { as } n \rightarrow \infty,
$$

thanks to (2.3). This says that the weak law of large numbers for $\left\{\Delta_{k, n}^{2}\right\}$ implies the central limit theorem for $\left\{X_{k, n}\right\}$.

Proof of Theorem 1.1. We consider the case $f_{n}=\tilde{K}_{n}$ only. Since \{\} in RHS of (2.1) is the difference of $\tilde{K}_{n}$ caused by changing only the state of $e_{k}$, we can see that $\left|\delta_{k} \tilde{K}_{n}\right| \leq 1$ and $\left|\Delta_{k, n}\right| \leq 1$, which proves (2.2).

Next we prove (2.3). Fix an integer $k \in\left\{1,2, \ldots, q_{n}\right\}$. We denote the set of the indices of edges which contain at least one endpoint of $e_{k}$ by $N\left(e_{k}\right)$. Let

$$
\begin{aligned}
D(k) & =\left\{\omega=\left(\omega_{1}, \ldots, \omega_{k}\right) ; \omega_{i}=1 \text { for } i \in N\left(e_{k}\right) \cap\{1, \ldots, k-1\} \text { and } \omega_{k}=0\right\}, \\
D^{\prime}(k) & =\left\{\omega^{\prime}=\left(\omega_{k+1}^{\prime}, \ldots, \omega_{q_{n}}^{\prime}\right) ; \omega_{i}^{\prime}=1 \text { for } i \in N\left(e_{k}\right) \cap\left\{k+1, \ldots, q_{n}\right\}\right\} .
\end{aligned}
$$

Noting that $\mathbb{T}^{d}$ has no cycles and $\left\{e_{1}, \ldots, e_{k}\right\}$ is connected, we can see that

$$
\delta_{k} \tilde{K}_{n}\left(\omega_{1}, \ldots, \omega_{k}, \omega_{k+1}^{\prime}, \ldots, \omega_{q_{n}}^{\prime}\right) \geq 0 \text { if } \omega \in D(k)
$$

and $\delta_{k} \tilde{K}_{n}\left(\omega_{1}, \ldots, \omega_{k}, \omega_{k+1}^{\prime}, \ldots, \omega_{q_{n}}^{\prime}\right)=p$ if $\omega \in D(k)$ and $\omega^{\prime} \in D^{\prime}(k)$. For any $\omega \in D(k)$,

$$
\begin{aligned}
\Delta_{k, n}\left(\omega_{1}, \ldots, \omega_{k}\right) & \geq \sum_{\omega^{\prime} \in D^{\prime}(k)} p P_{p}\left\{\left(\omega_{k+1}, \ldots, \omega_{q_{n}}\right)=\left(\omega_{k+1}^{\prime}, \ldots, \omega_{q_{n}}^{\prime}\right)\right\} \\
& \geq p \cdot p^{2 d}(1-p)=p^{2 d+1}(1-p) .
\end{aligned}
$$


Thus we have

$$
\begin{aligned}
E_{p} \Delta_{k, n}^{2} & \geq E_{p}\left[\left\{\Delta_{k, n}\left(\omega_{1}, \ldots, \omega_{k}\right)\right\}^{2} ; D(k)\right] \\
& \geq\left\{p^{2 d+1}(1-p)\right\}^{2} \cdot p^{2 d}=p^{6 d+2}(1-p)^{2} \equiv \sigma(p) .
\end{aligned}
$$

Finally we verify (2.4). We write $e=\left\langle x_{1}(e), x_{2}(e)\right\rangle$ when $d\left(O, x_{1}(e)\right)<$ $d\left(O, x_{2}(e)\right)$. For $e_{1}, e_{2} \in E$, let $d\left(e_{1}, e_{2}\right)=\min _{i, j=1,2} d\left(x_{i}\left(e_{1}\right), x_{j}\left(e_{2}\right)\right)$. Since there are no cycles on $\mathbb{T}^{d}$, we can see that $\delta_{k} \tilde{K}_{n}$ depends only on the state of $N\left(e_{k}\right)$. So $\Delta_{i, n}$ and $\Delta_{j, n}$ are independent if $d\left(e_{i}, e_{j}\right)>1$. Now (2.4) easily follows from Chebyshev's inequality.

\section{§3. Proof for Sierpiński Carpet Lattices}

We shall prove Theorem 1.2 (i) in $\S 3.1$ and (ii) in $\S 3.2-3.5$.

Let $L \geq 2$. Fix $T \subset \mathbf{T}_{L}$ which satisfies (1.1), (1.2) and (1.3).

We prepare some notations. In the same way as in $\S 2$, we define $E_{T}=$ $\left\{e_{1}, e_{2}, \ldots\right\}, q_{n},\left\{\mathcal{F}_{k}\right\}, \Delta_{k, n}$ and $X_{k, n}$. For $\underline{x}=\left(x_{1}, x_{2}\right) \in \mathbb{Z}_{+}^{2} \equiv\{0,1,2, \ldots\}^{2}$, we define $G_{m}^{T}(\underline{x})=\left(V_{m}^{T}(\underline{x}), E_{m}^{T}(\underline{x})\right)$ with

$$
\begin{aligned}
& V_{m}^{T}(\underline{x})=V_{T} \cap\left\{\left[x_{1} L^{m},\left(x_{1}+1\right) L^{m}\right] \times\left[x_{2} L^{m},\left(x_{2}+1\right) L^{m}\right]\right\}, \\
& E_{m}^{T}(\underline{x})=\left\{\langle u, v\rangle \in E_{T} ; u, v \in V_{m}^{T}(\underline{x})\right\} .
\end{aligned}
$$

Let $m<n$. For $e \in E_{T}$, let $\underline{x}_{m}(e)=\left(x_{1}^{m}(e), x_{2}^{m}(e)\right) \in \mathbb{Z}_{+}^{2}$ be such that $e$ belongs to $G_{m}^{T}(e) \equiv G_{m}^{T}\left(\underline{x}_{m}(e)\right)$. For $\underline{x}=\left(x_{1}, x_{2}\right) \in \mathbb{Z}_{+}^{2}$, let $\|\underline{x}\|_{1}=\left|x_{1}\right|+\left|x_{2}\right|$ and $\|\underline{x}\|_{\infty}=\max \left\{\left|x_{1}\right|,\left|x_{2}\right|\right\}$. When we regard $G_{n}$ as a union of $G_{m}$ 's, we often index these $G_{m}$ 's by $T^{n-m}$. For fixed $m$, we identify an element of $T^{n-m}$ as that of $\mathbb{Z}_{+}^{2}$ in obvious fashion.

For a region $S$ of $G_{T}$, the border points of $S$ is defined by the inner boundary sites when we regard $S$ as a subset of $\mathbb{Z}^{2}$. For $m>0$, int $G_{m}$ denotes the graph obtained by deleting the edges connecting the border points of $G_{m}$. For $m>l>0$, let

$$
\partial_{l} G_{m}=\left\{G_{l}(\underline{x}): \underline{x} \in T^{m-l}, G_{l}(\underline{x}) \text { contains some of border points of } G_{m}\right\}
$$

(see Figure 4). We often use the following facts.

Lemma 3.1. (i) For $m>l>0,\left\|G_{m}\right\| \geq|T|^{m-l} \times \|$ int $G_{l} \|$.

(ii) $\|$ int $G_{m}\|/\| G_{m} \| \rightarrow 1$ as $m \rightarrow \infty$.

(iii) $\sup _{l \geq 1} \frac{\left\|\partial_{l} G_{l+j}\right\|}{\left\|G_{l+j}\right\|} \rightarrow 0$ as $j \rightarrow \infty$. 


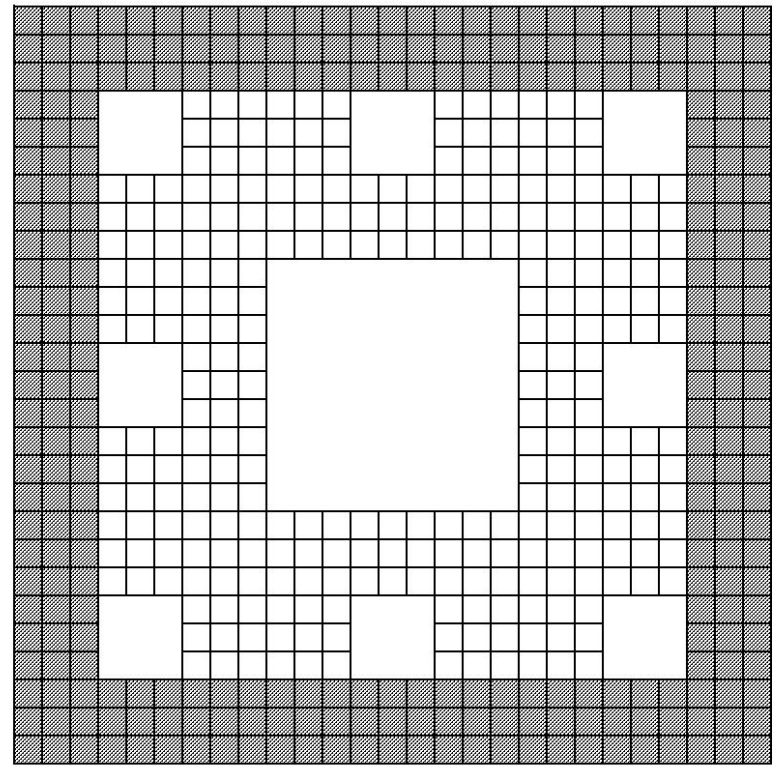

Figure 4. The shaded region indicates $\partial_{1} G_{3}$.

Proof. (i) is obvious. By (1.3), we have $|T| \geq 4(L-1)$ and $\|$ int $G_{1} \| \geq 4 L$. Noting that $\left\|\operatorname{int} G_{m}\right\|=\left\|G_{m}\right\|-4 L^{m}$ and $\left\|G_{m}\right\| \geq\{4(L-1)\}^{m-1} \cdot 4 L$, we can prove (ii). Using (i) and (ii), we have

$$
\sup _{l \geq 1} \frac{\left\|\partial_{l} G_{l+j}\right\|}{\left\|G_{l+j}\right\|} \leq \frac{4\left(L^{j}-1\right)}{\{4(L-1)\}^{j}} \sup _{l \geq 1} \frac{\left\|G_{l}\right\|}{\left\|\operatorname{int} G_{l}\right\|} \rightarrow 0 \text { as } j \rightarrow \infty,
$$

which proves (iii).

\section{§3.1. Proof of Theorem 1.2 (i)}

For a finite subset $S$ of $G_{T}, K(S)$ denotes the number of open clusters in $S$. Suppose that $n>m>0$. Noting that $K_{n} \leq \sum_{\underline{x} \in T^{n-m}} K\left(G_{m}^{T}(\underline{x})\right)$ and $E_{p} K\left(G_{m}^{T}(\underline{x})\right)=E_{p} K_{m}$ for any $\underline{x} \in T^{n-m}$, we have $E_{p} K_{n} \leq|T|^{n-m} E_{p} K_{m}$. Dividing by $q_{n} \equiv\|B(n)\|$ and using Lemma 3.1 (i), we can see that

$$
\frac{E_{p} K_{n}}{q_{n}} \leq \frac{|T|^{n-m} E_{p} K_{m}}{q_{n}} \leq \frac{E_{p} K_{m}}{\|\operatorname{int} B(m)\|}=\frac{E_{p} K_{m}}{q_{m}} \frac{q_{m}}{\|\operatorname{int} B(m)\|} .
$$


This implies the existence of the limit of $E_{p} K_{n} / q_{n}$.

Let $E_{n}=\left\{\left|\left(K_{n}-E_{p} K_{n}\right) / q_{n}\right| \geq|T|^{-n / 4}\right\}$. Noting that $\left|\Delta_{k, n}^{2}\right| \leq 1$, we have

$$
E_{p}\left(\frac{K_{n}-E_{p} K_{n}}{q_{n}}\right)^{2}=\frac{\operatorname{var}_{p} K_{n}}{q_{n}^{2}}=\frac{1}{q_{n}^{2}} \sum_{k=1}^{q_{n}} E_{p} \Delta_{k, n}^{2} \leq \frac{1}{q_{n}} .
$$

It follows from Chebyshev's inequality and Lemma 3.1 (i) that

$$
P\left(E_{n}\right) \leq|T|^{n / 2} \cdot \frac{1}{q_{n}} \leq \frac{|T|^{n / 2}}{4 L|T|^{n-1}}=\frac{1}{4 L|T|^{n / 2-1}} .
$$

By Borel-Cantelli's lemma, we can show the almost sure convergence of $K_{n} / q_{n}$.

\section{§3.2. Proof of Theorem 1.2 (ii): preliminary}

We quote some results in [4], which we need later.

Lemma 3.2 ([4]). Let $L \geq 2$. Suppose that $T \subset \mathbf{T}_{L}$ satisfies (1.1), (1.2) and (1.3).

(i) We have $0<p_{s} \leq p_{c} \leq p_{s}^{*}<1$.

(ii) When $p<p_{s}$, there exist $n_{0} \in \mathbb{N}, \theta<1$ and $C>0$ such that

$$
Q_{n_{0}+m,[1,3 L]}(p) \leq C \theta^{2^{m}} \text { for all } m \geq 0 \text {. }
$$

(iii) (an $R S W$-type lemma) For $k \geq m \geq 2$,

$$
\limsup _{n \rightarrow \infty} Q_{n,[m, m]}(p)=1 \Longleftrightarrow \limsup _{n \rightarrow \infty} Q_{n,[k, m]}(p)=1,
$$

which also holds for dual crossing probabilities.

We can easily check the condition (2.2) for $f_{n}=K_{n}$ or $\tilde{K}_{n}$ as in $\S 2$. While we can verify (2.3) for $K_{n}$ by using the FKG inequality as in [9], we cannot apply this method to $\tilde{K}_{n}$. So we prove $(2.3)$ for $\tilde{K}_{n}$ by the same argument as in $\S 2$. Fix an integer $k \in\left\{1,2, \ldots, q_{n}\right\}$. Let $\bar{N}\left(e_{k}\right)=\left\{i \in\left\{1,2, \ldots, q_{n}\right\} ; e_{i} \in\right.$ $E_{n}^{T} \backslash N\left(e_{k}\right)$ and $e_{i} \cap e_{j} \neq \emptyset$ for some $\left.j \in N\left(e_{k}\right)\right\}$. Note that $\left\|N\left(e_{k}\right)\right\| \leq 7$ and $\left\|\bar{N}\left(e_{k}\right)\right\| \leq 16$. We modify the definitions of $D(k)$ and $D^{\prime}(k)$ :

$$
\begin{aligned}
& D(k)=\left\{\omega=\left(\omega_{1}, \ldots, \omega_{k}\right) ; \begin{array}{c}
\omega_{i}=1 \text { for } i \in N\left(e_{k}\right) \cap\{1, \ldots, k-1\}, \omega_{k}=0, \\
\omega_{j}=0 \text { for } j \in \bar{N}\left(e_{k}\right) \cap\{1, \ldots, k-1\}
\end{array}\right\}, \\
& D^{\prime}(k)=\left\{\omega^{\prime}=\left(\omega_{k+1}^{\prime}, \ldots, \omega_{q_{n}}^{\prime}\right) ; \begin{array}{l}
\omega_{i}^{\prime}=1 \text { for } i \in N\left(e_{k}\right) \cap\left\{k+1, \ldots, q_{n}\right\}, \\
\omega_{j}^{\prime}=0 \text { for } j \in \bar{N}\left(e_{k}\right) \cap\left\{k+1, \ldots, q_{n}\right\}
\end{array}\right\} .
\end{aligned}
$$

Now we can prove $(2.3)$ for $\tilde{K}_{n}$ along the same line as in $\S 2$.

The condition (2.4) will be checked in $\S 3.3-3.5$. We shall give a proof only for $K_{n}$. Almost the same proof works for $\tilde{K}_{n}$. 


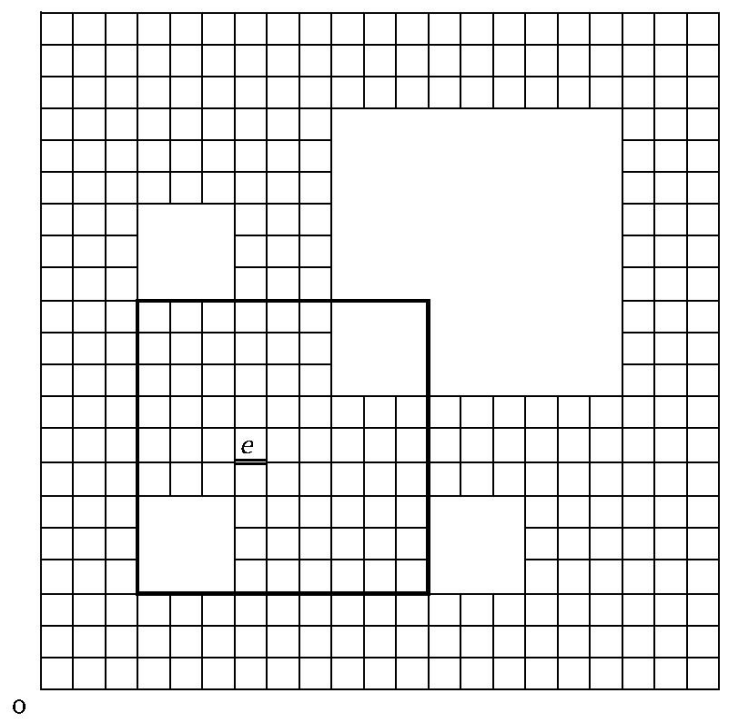

Figure 5. The thick-lined box is $\bar{G}_{1, \cdot}^{T}(e)$.

\section{§3.3. $\quad$ CLT for subcritical regime $: p<p_{s}$}

Let $n>m$. We define $\bar{G}_{m, n}^{T}(e)=\left(\bar{V}_{m, n}^{T}(e), \bar{E}_{m, n}^{T}(e)\right)$ by $\bar{V}_{m, n}^{T}(e)$

$=V_{n}^{T} \cap\left\{\left[\left(x_{1}^{m}(e)-1\right) L^{m},\left(x_{1}^{m}(e)+2\right) L^{m}\right] \times\left[\left(x_{2}^{m}(e)-1\right) L^{m},\left(x_{2}^{m}(e)+2\right) L^{m}\right]\right\}$, $\bar{E}_{m, n}^{T}(e)=\left\{\langle u, v\rangle \in E_{n}^{T} ; u, v \in \bar{V}_{m, n}^{T}(e)\right\}$

(see Figure 5). The (inner) boundary of $\bar{G}_{m, n}^{T}(e)$ is defined by

$$
\left\{u \in \bar{V}_{m, n}^{T}(e) ;\langle u, v\rangle \in E_{T} \text { for some } v \in V_{n}^{T} \backslash \bar{V}_{m, n}^{T}(e)\right\} .
$$

For $e \in E_{n}^{T}$, let

$$
D(e, m, n)=\left\{\begin{array}{c}
\text { each endpoints of edge } e \text { belong to } \\
\text { different open clusters in } \bar{G}_{m, n}^{T}(e) \\
\text { which are connected to the boundary of } \bar{G}_{m, n}^{T}(e)
\end{array}\right\} .
$$

Noting that $P_{p}(D(e, m, n)) \leq 4 Q_{m,[1,3]}$, we can see the following by Lemma 3.2 (ii). 
Lemma 3.3. If $p<p_{s}$, then for given $\varepsilon>0$ we can take a sufficiently large $m_{0}$ such that $P_{p}(D(e, m, n)) \leq \varepsilon$ for all $n>m \geq m_{0}$ and $e \in E_{n}^{T}$.

Now we prove Theorem 1.2 (ii) for $p<p_{s}$. We shall verify (2.4). Fix $\varepsilon>0$. We take sufficiently large $m$ so that the statement of Lemma 3.3 holds. Let $n>m$. We compare $\Delta_{k, n}$ with $\Delta_{k, n}^{\prime}={ }_{m} \Delta_{k, n}^{\prime} \equiv \Delta_{k, n} 1_{D\left(e_{k}, m, n\right)^{c}}$. We have

$$
\begin{aligned}
& \frac{1}{q_{n}}\left|\sum_{k=1}^{q_{n}}\left(\Delta_{k, n}^{2}-E_{p} \Delta_{k, n}^{2}\right)\right| \\
& \leq \frac{1}{q_{n}}\left|\sum_{k=1}^{q_{n}}\left\{\Delta_{k, n}^{2}-\left(\Delta_{k, n}^{\prime}\right)^{2}\right\}\right|+\frac{1}{q_{n}}\left|\sum_{k=1}^{q_{n}}\left\{\left(\Delta_{k, n}^{\prime}\right)^{2}-E_{p}\left(\Delta_{k, n}^{\prime}\right)^{2}\right\}\right| \\
& \quad+\frac{1}{q_{n}}\left|\sum_{k=1}^{q_{n}}\left\{E_{p}\left(\Delta_{k, n}^{\prime}\right)^{2}-E_{p} \Delta_{k, n}^{2}\right\}\right|=S_{\mathrm{I}}+S_{\mathrm{II}}+S_{\mathrm{III}} .
\end{aligned}
$$

Noting that $\left|\Delta_{k, n}\right| \leq 1$ and $E_{p}\left|\Delta_{k, n}-\Delta_{k, n}^{\prime}\right| \leq P_{p}\left(D\left(e_{k}, m, n\right)\right)$, we have

$$
\begin{aligned}
E_{p}\left(S_{\mathrm{I}}\right) & \leq \frac{1}{q_{n}} \sum_{k=1}^{q_{n}} E_{p}\left|\Delta_{k, n}+\Delta_{k, n}^{\prime}\right|\left|\Delta_{k, n}-\Delta_{k, n}^{\prime}\right| \\
& \leq \frac{1}{q_{n}} q_{n} \cdot 2 \cdot \varepsilon=2 \varepsilon .
\end{aligned}
$$

Similarly, we have $E_{p}\left(S_{\mathrm{III}}\right) \leq \frac{1}{q_{n}} \sum_{k=1}^{q_{n}} E_{p}\left|\left(\Delta_{k, n}^{\prime}\right)^{2}-\Delta_{k, n}^{2}\right| \leq 2 \varepsilon$.

Next we show that $E_{p}\left(S_{\mathrm{II}}^{2}\right) \rightarrow 0$ as $n \rightarrow \infty$. To this end, we shall prove $\Delta_{i, n}^{\prime}$ depends on the states of the edges in $\bar{G}_{m, n}^{T}\left(e_{i}\right)$, so that $\Delta_{i, n}^{\prime}$ and $\Delta_{j, n}^{\prime}$ are independent if $\left\|\underline{x}_{m}\left(e_{i}\right)-\underline{x}_{m}\left(e_{j}\right)\right\|_{\infty}>3$. We split $D\left(e_{i}, m, n\right)^{c}$ into two disjoint events:

$$
\begin{aligned}
& G\left(e_{i}, m, n\right)=\left\{\begin{array}{c}
\text { in } \bar{G}_{m, n}^{T}\left(e_{i}\right), \text { endpoints of } e_{i} \text { is connected to each other } \\
\text { by an open path not traversing } e_{i}
\end{array}\right\}, \\
& H\left(e_{i}, m, n\right)=\left\{\begin{array}{c}
\text { each endpoints of edge } e_{i} \text { belong to different } \\
\text { open clusters, but not both of these clusters are } \\
\text { connected to the boundary of } \bar{G}_{m, n}^{T}\left(e_{i}\right)
\end{array}\right\} .
\end{aligned}
$$

When $G\left(e_{i}, m, n\right)$ occurs, the number of open clusters are independent of the state of $e_{i}$. Hence $\delta_{i} K_{n} 1_{G\left(e_{i}, m, n\right)}=0$. On the other hand, we can see that

$$
\delta_{i} K_{n} 1_{H\left(e_{i}, m, n\right)}= \begin{cases}-(1-p) & \text { if } \omega_{i}=1 \\ p & \text { if } \omega_{i}=0\end{cases}
$$


Thus $\Delta_{i, n}^{\prime}$ is measurable with respect to the states of edges in $\bar{G}_{m, n}^{T}\left(e_{i}\right)$. Now we have

$$
\begin{aligned}
E_{p}\left(S_{\mathrm{II}}^{2}\right) & =\frac{1}{q_{n}^{2}} \sum_{i=1}^{q_{n}} \sum_{j=1}^{q_{n}} \operatorname{cov}_{p}\left(\left(\Delta_{i, n}^{\prime}\right)^{2},\left(\Delta_{j, n}^{\prime}\right)^{2}\right) \\
& \leq \frac{1}{q_{n}^{2}} \sum_{i=1}^{q_{n}} \sum_{j:\left\|\underline{x}_{m}\left(e_{j}\right)-\underline{x}_{m}\left(e_{i}\right)\right\|_{\infty} \leq 3} 4 \leq \frac{1}{q_{n}^{2}} q_{n} \cdot 49 \cdot 4=\frac{196}{q_{n}} .
\end{aligned}
$$

Using Markov's and Chebyshev's inequalities, we can prove (2.4). This completes the proof.

\section{§3.4. CLT for critical regime $: p \in\left[p_{s}, p_{s}^{*}\right]$}

Once we prove the following lemma, we can obtain the CLT for $p \in\left[p_{s}, p_{s}^{*}\right]$ by the same argument in $\S 3.3$.

Lemma 3.4. We assume that (1.4) holds. If $p \leq p_{s}^{*}$, then for given $\varepsilon>0$ we can take a sufficiently large $m_{0}$ such that $P_{p}(D(e, m, n)) \leq \varepsilon$ for all $n>m \geq m_{0}$ and $e \in E_{n}^{T}$.

Proof. Since $T=\mathbf{T}_{2}$ (i.e. $G_{T}=\mathbb{Z}^{2}$ ) is the only case that $T \subset \mathbf{T}_{2}$ satisfies (1.1), (1.2) and (1.3), we consider the case $L \geq 3$. Using the duality equation, (1.4) and Lemma 3.2 (iii), we can prove that there exists a positive constant $\delta$ such that $Q_{n,[3 L, 1]}^{*} \geq \delta$ for all $n$. For $e \in E_{T}$ and $i \geq 1$, let $D_{i}(e)$ be the union of four $G_{i}$ 's (or $L^{i} \times L^{i}$ holes), which are connected to the corner closest to $e$ among the corners of $G_{i}^{T}(e)$. Let $A_{i}(e)$ be $G_{i-1}$ 's (or $L^{i-1} \times L^{i-1}$ holes) which contain the border points of $D_{i}(e)$. Note that for all $i$ there is an dual closed circuit in $A_{i}(e)$ with probability $\geq \delta^{4}$. We take $m_{0}$ such that $\left(1-\delta^{4}\right)^{m_{0}} \leq \varepsilon$. For $m>m_{0}$, we have

$$
\begin{aligned}
& P_{p}(D(e, m, n)) \\
& \leq P_{p}\left(\bigcap_{i=2, \ldots, m_{0}+1}\left\{\text { there is no dual closed circuit in } A_{i}(e)\right\}\right) \\
& \leq\left(1-\delta^{4}\right)^{m_{0}} \leq \varepsilon .
\end{aligned}
$$

This completes the proof. 


\section{§3.5. CLT for supercritical regime $: p>p_{s}^{*}$}

First we note that by Lemma 3.1, for given $\eta>0$ we can find $j_{0}$ such that $\left\|\partial_{l} G_{l+j}\right\| / \|$ int $G_{l+j} \| \leq \eta$ for all $l \geq 1$ and $j \geq j_{0}$.

Lemma 3.5. Suppose that $p>p_{s}^{*}$. For fixed $j$, we have $\alpha(l)=\alpha(l, p) \equiv P_{p}\left(\right.$ There exists an open circuit in $\left.\partial_{l} G_{l+j}\right) \rightarrow 1$ as $l \rightarrow \infty$.

Proof. By the FKG inequality, we have

$$
\alpha(p) \geq\left[Q_{l,\left[L^{j}, 1\right]}(p)\right]^{4} \geq\left[\left\{Q_{l,[3,1]}(p)\right\}^{N_{j}}\right]^{4}, \text { where } N_{j}=2\left\lceil\left(L^{j}+1\right) / 3\right\rceil-1 .
$$

Since $\lim \sup Q_{l,[3,1]}(p)=1$ for $p>p_{s}^{*}$, we get the conclusion.

Now we check (2.4). We fix an integer $l$. Suppose that $n>m>l+j_{0}$. We regard $B(n)=G_{n}^{T}$ as $\bigcup_{\underline{x} \in T^{n-m}} G_{m}^{T}(\underline{x})$. For $\underline{x} \in T^{n-m}$, let $1(\underline{x})$ be the indicator function of $\left\{\right.$ there exists an open circuit in $\left.\partial_{l} G_{m}(\underline{x})\right\}$. Let $S_{k}=\Delta_{k, n}^{2}-E_{p} \Delta_{k, n}^{2}$ and $\tilde{S}_{k}=S_{k} 1\left(\underline{x}_{m}\left(e_{k}\right)\right)$. Note that $\left|\tilde{S}_{k}\right| \leq\left|S_{k}\right| \leq 1$ and $\left|E_{p} \tilde{S}_{k}\right|=\left|E_{p}\left(S_{k}-\tilde{S}_{k}\right)\right|=$ $\left|E_{p} S_{k}\left(1-1\left(\underline{x}_{m}\left(e_{k}\right)\right)\right)\right| \leq 1-\alpha(l)$. For $i, k \in\left\{1,2, \ldots, q_{n}\right\}$, we have

$$
\begin{aligned}
E_{p}\left[S_{i} S_{k}\right] & =E_{p}\left[\tilde{S}_{i} \tilde{S}_{k}\right]+E_{p}\left[S_{i}\left(S_{k}-\tilde{S}_{k}\right)\right]+E_{p}\left[\tilde{S}_{k}\left(S_{i}-\tilde{S}_{i}\right)\right] \\
& \leq E_{p}\left[\tilde{S}_{i} \tilde{S}_{k}\right]+2(1-\alpha(l)) .
\end{aligned}
$$

Let $U(n)=\bigcup_{\underline{x} \in T^{n-m}} \partial_{l} G_{m}(\underline{x})$. Note that by the choice of $m$ and Lemma 3.1 (i),

$$
\|U(n)\| \leq \sum_{\underline{x} \in T^{n-m}}\left\|\partial_{l} G_{m}(\underline{x})\right\| \leq \sum_{\underline{x} \in T^{n-m}} \eta\left\|\operatorname{int} G_{m}(\underline{x})\right\| \leq \eta q_{n}
$$

Let $I_{n}=\left\{(i, k) ; 1 \leq i, k \leq q_{n}\right\}$ and $\hat{I}_{n}=\left\{(i, k) ; e_{i}, e_{k} \notin U(n)\right.$ and $\| \underline{x}_{m}\left(e_{i}\right)-$ $\left.\underline{x}_{m}\left(e_{k}\right) \|_{1} \geq 2\right\}$. Note that $\left|I_{n} \backslash \hat{I}_{n}\right| \leq 2 q_{n}|| U(n) \|+q_{n} \cdot 5 q_{m} \leq 2 \eta q_{n}^{2}+5 q_{m} q_{n}$. By the same argument as in $\S 3.3$, we can see that if $(i, k) \in \hat{I}_{n}$, then $\tilde{S}_{i}$ and $\tilde{S}_{k}$ are independent and $E_{p}\left[\tilde{S}_{i} \tilde{S}_{k}\right]=E_{p}\left[\tilde{S}_{i}\right] E_{p}\left[\tilde{S}_{k}\right] \leq(1-\alpha(l))^{2}$. Thus we have

$$
\begin{aligned}
\frac{1}{q_{n}^{2}} \sum_{(i, k) \in I_{n}} E_{p}\left[S_{i} S_{k}\right] & \leq \frac{1}{q_{n}^{2}}\left(\left|\hat{I}_{n}\right| \cdot(1-\alpha(l))^{2}+\left|I_{n} \backslash \hat{I}_{n}\right| \cdot 1+q_{n}^{2} \cdot 2(1-\alpha(l))\right) \\
& \leq(1-\alpha(l))^{2}+\left(2 \eta+\frac{5 q_{m}}{q_{n}}\right)+2(1-\alpha(l)) .
\end{aligned}
$$


For fixed $l$ and $m$ we have

$$
\limsup _{n \rightarrow \infty} \frac{1}{q_{n}^{2}} E_{p}\left\{\sum_{k=1}^{q_{n}}\left(\Delta_{k, n}^{2}-E_{p} \Delta_{k, n}^{2}\right)\right\}^{2} \leq(1-\alpha(l))^{2}+2 \eta+2(1-\alpha(l)) .
$$

Letting $\eta \searrow 0$ and $l \rightarrow \infty$, we get the desired result by Lemma 3.5.

\section{Acknowledgements}

This is part of the second author's thesis. The authors would like to thank Professor Yasunari Higuchi for his advice and encouragement. They also thank Professor Rahul Roy, Professor Hideki Tanemura and Professor Masato Shinoda for discussions.

\section{References}

[1] Aizenman, M., Chayes, J. T., Chayes, L., Fröhrich, J. and Russo, L., On a sharp transition from area law to perimeter law in a system of random surfaces, Comm. Math. Phys., 92 (1983), 19-69.

[2] Grimmett, G. R., Percolation, second edition, Grundlehren der mathematischen Wissenschaften, Springer, 321, 1999.

[3] Häggström, O., Invariant percolation on trees and the mass-transport method, Bulletin of the International Statistical Institute, 52nd Session Proceedings, Tome LVIII, Book 1, (1999), 363-366.

[4] Kumagai, T., Percolation on pre-Sierpinski carpets, in: Elworthy, K. D., Kusuoka, S., Shigekawa, I. eds., New Trend in Stochastic Analysis, (1997), 288-304, World-Scientific.

[5] McLeish, D. L., Dependent central limit theorems and invariance principles, Ann. Probab., 2 (1974), 620-628.

[6] Penrose, M. D., A central limit theorem with applications to percolation, epidemics and boolean models, Ann. Probab., 29 (2001), 1515-1546.

[7] Shinoda, M., Existence of phase transition of percolation on Sierpiński carpet lattices, J. Appl. Probab., 39 (2002), 1-10.

[8] Wu, X. Y., Uniqueness of infinite open cluster for high-density percolation on lattice Sierpinski carpet, Acta Math. Sin. (Engl. Ser.), 17 (2001), 141-146.

[9] Zhang, Y., A martingale approach in the study of percolation clusters on the $Z^{d}$ lattice, J. Theoret. Probab., 14 (2001), 165-187. 